\title{
Endogenous Jaagsiekte Sheep Retrovirus RNA is expressed by different cell types in ovine foetus and placenta
}

\author{
E. Sanna ${ }^{1}$, M.P. Sanna ${ }^{1}$, C. Loddo ${ }^{1}$, L. Sanna ${ }^{1}$, M. Mura ${ }^{1,2}$, T. Cadelano ${ }^{1}$, and A. Leoni ${ }^{1}$
}

'Istituto di Patologia Generale, Anatomia Patologica e Clinica Ostetrico-Chirurgica Veterinaria, Facoltà di Veterinaria, Università di Sassari, Via Vienna 2, 07100, Sassari, Italy and ${ }^{2}$ Department of Medical Microbiology and Parasitology, College of Veterinary Medicine, University of Georgia, Athens, USA

Accepted: 13/05/02

Key words: enJSRV, RNA, sheep, foetus, placenta

\section{SUMMARY}

The endogenous retroviruses are inherited elements transmitted trough the germline of most animal species and their biological role is still controversial. Ovine Pulmonary Carcinoma (OPC) represents a good model for studying the interactions of endogenous retroviruses with their exogenous counterparts. The type D exogenous retrovirus known as Jaagsiekte Sheep Retro-Virus (JSRV) is necessary and sufficient to cause OPC in domestic and wild sheep, but both affected and unaffected animals host in their genome 15 to 20 copies of related endogenous retroviruses named endogenous JSRV (enJSRV). In this study we evaluated the expression of enJSRV gag sequences in ovine foetal and placental tissues. RNA in situ hybridisation was performed on tissue sections of thymi, lymph nodes and lungs from ovine foetuses and related placentas, taken at a late stage of development. Reverse transcriptase-in situ polymerase chain reactions were also carried out on placental samples to better define the involved cells. In foetal tissues, specific signals were observed in the thymus medulla, lymph nodes and, at a lesser extent, in foetal bronchiolar cells. In the placental tissues, positive areas were detected in various cell types in the sincythium-and cyto-trophoblast. These data demonstrate that en JSRV RNA is largely expressed in a broad spectrum of cells including tissues which are critical for the development of the immune system.

\section{INTRODUCTION}

The endogenous retroviruses (ERVs) are present in the genome of most animal species as a consequence of ancient infections by exogenous counterparts, followed by deletions or point mutations with loss of replication activity. They are vertically transmitted and their biological relevance is under discussion, as both harmful and beneficial effects to the host have been envisaged (Löewer $e t$ al., 1996; Larsson and Anderson, 1998). In human tissues, by means of RNA in situ hybridisation, consistent Human Endogenous Retro-Virus K (HERV-K) expression of gag and env genes was found to be common to all testicular and ovarian germ cell tumours (GCTs) (Herbst et al., 1996) and these findings supported the concept of an involvement and a common pathogenic behaviour 
of HERV-K in some human malignant tumours. In other studies, the Endogenous Retro-Virus 3 (ERV-3) expression appeared to be upregulated at the inflammatory sites, thus suggesting a role in the pathogenesis of some diseases (Katsumata, 1997). In general, it is accepted that the possible effects of the ERVs include the production of somatic or germline mutations with loss of tumour suppression activity (Taruscio and Mantovani, 1998), an influence on adjacent cellular genes (Feuchter and Mager, 1990, 1992), and an involvement of their products in immunosuppressive or autoimmune mechanisms (Krieg et al., 1989; Nakagawa and Harrison, 1996). On the other hand, some endogenous retrovirus-like elements may also play protective roles against exogenous retroviruses (Best et al., 1996).

Ovine Pulmonary Carcinoma (OPC) represents a good model for studying the interactions of the endogenous retroviruses with their exogenous counterparts. An exogenous type-D beta-retrovirus known as Jaagsiekte Sheep Retro-Virus (JSRV) was found to be necessary and sufficient to cause OPC (Palmarini et al., 1999), but both OPC-affected and unaffected sheep host 15 to 20 copies of endogenous retroviruses (enJSRVs), which are highly related to JSRV but yet distinct (Hecht et al., 1994, 1996; Bai et al., 1996). From an evolutionary point of view, enJSRVs are rather young and virtually full-length retroviruses, and this is consistent with the existence of a closely related exogenous counterpart. They are transcriptionally active in various tissues of adult unaffected sheep, but unable to replicate as a result of defects located in the gag gene (Palmarini et al., 2000b). In OPCaffected sheep and moufflons, exogenous JSRV has been detected in tumour cells, normal type II pneumocytes, macrophages in alveoli near the lesions, and in mediastinal lymph-nodes (Palmarini et al., 1995, 1996a; Holland et al., 1999; Sanna et al., 2001), whilst enJSRVs RNA were found in the luminal and glandular epithelium of the uterus, and, at a lesser extent, in the gut and bronchial epithelium (Palmarini et al., 2000b). It has recently been demonstrated that progesterone levels influence the transcriptional activity of enJSRV long terminal repeats in the endometrial epithelia of the ovine uterus, and this might support a role for enJSRVs in conceptus-endometrial interactions during the peri- implantation period and early placental morphogenesis (Palmarini et al., 2001).

With the aim to evaluate the expression of enJSRV in foetal and placental tissues, we have developed in situ hybridisation and in situ reverse transcriptase PCR (IS-RT-PCR) protocols specific for the detection of endogenous RNA sequences.

\section{MATERIALS AND METHODS}

\section{Samples}

Thymus, mesenteric lymph nodes and lung samples were collected from n. 4 ovine foetuses at a late stage of development (last week of pregnancy, length ranging from 42 to $48 \mathrm{~cm}$ ). The related placentas were also taken and all the tissues were fixed with $4 \%$ paraformaldeheyde for $23 \mathrm{hrs}$, paraffin wax-embedded and cut at $5 \mu \mathrm{m}$.

\section{In situ hybridisation}

All stages of the protocols were performed with a "capillary gap" equipment (Microprobe, Biomeda, Foster City, CA, USA) and all the reagents were added with $0.1 \%$ diethyl-pyro-carbonate (DEPC) in order to prevent bacterial contamination. The sections, mounted on capillary gap slides (Probe-On Plus, Biomeda), were de-waxed, rehydrated, treated with $0.2 \mathrm{~N} \mathrm{HCl}$ for $15 \mathrm{~min}$ (room temperature; RT), $0.3 \%$ Triton $\mathrm{X}-100$ for $15 \mathrm{~min}$ (RT), protease $\mathrm{K}\left(+2 \mathrm{mM} \mathrm{CaCl}_{2}\right) 5 \mu \mathrm{g} / \mathrm{ml}$ for 10 min at $37^{\circ} \mathrm{C}$ and $10 \mathrm{mM}$ Tris- $\mathrm{HCl} \mathrm{pH} 8.3$ for $5 \mathrm{~min}$ (RT) and post-fixed with $4 \%$ paraformaldeheyde for $5 \mathrm{~min}$ (RT). After phosphate-buffered saline (PBS) and sterile water washings, the samples were treated with an aqueous solution containing $0.1 \mathrm{M}$ tri-ethanolamine (TEA) and $0.25 \%$ acetic anhydride for $10 \mathrm{~min}$ (RT) and then washed with PBS. In order to remove the DNA, the sections underwent a treatment with a solution containing $280 \mu l$ Rnase-free Dnase I 10U/ $\mu l$ in $720 \mu l$ Dnase buffer (20mM Tris- $\mathrm{HCl}, 10 \mathrm{mM} \mathrm{MgCl}_{2}$ at $\mathrm{pH}$ 8) for $25 \mathrm{~min}$ at $37^{\circ} \mathrm{C}$. The samples were washed with PBS and the enzyme was denatured with a treatment at $80^{\circ} \mathrm{C}$ for $5 \mathrm{~min}$. A pre-hybridisation step was performed with a cocktail consisting of 2 $\mathrm{x}$ saline-sodium-phosphate-EDTA (SSPE; Sigma Chemical Co., St. Louis, USA) with $50 \%$ formamide for $1 \mathrm{~h}$ at $37^{\circ} \mathrm{C}$. 
The slides were then hybridised with two digoxygenin (DIG)-labelled oligonucleotides specific for the gag gene (Sarda Bioanalysis, Cagliari, Italy), each diluted at a $0,1 \mathrm{MM}(0,95 \mathrm{ng} / \mu \mathrm{l})$ concentration in Fluka hybridisation solution IV (Fluka ChemikaBiochemika, Buchs, Switzerland) with $50 \%$ formamide (5' - CAATGCCTTGCATGTAGGAGGGTC CAATGTC-3', and 5' - TCTTGTTCCGGGCTTGCTGCT GGAAAAGTAC-3').

Slides were warmed to $80^{\circ} \mathrm{C}$ for $3 \mathrm{~min}$ and hybridisation was performed overnight at $36^{\circ} \mathrm{C}$. The samples were then washed as follows: $5 \mathrm{x}$ SSPE with $50 \%$ formamide at $36^{\circ} \mathrm{C}$ for $15 \mathrm{~min}$, twice; 2x SSPE repeatedly; 2x SSPE for $20 \mathrm{~min}$; $0.2 \mathrm{x}$ SSPE at $53^{\circ} \mathrm{C}$ for $10 \mathrm{~min}$, twice; $0.1 \mathrm{x}$ SSPE repeatedly; 0.1X SSPE for $5 \mathrm{~min}$; buffer $1(0.1 \mathrm{M}$ Tris, $0.15 \mathrm{M} \mathrm{NaCl}, \mathrm{pH}$ 7.6) for $5 \mathrm{~min}$. The slides were then treated with a solution containing Normal Rat Serum (NRS) $20 \mu \mathrm{l}, 0.3 \%$ Triton X-100 3 $\mu l$, diluted in $1 \mathrm{ml}$ of buffer 1 for $30 \mathrm{~min}$. Signal was detected by using the Dig-Nucleic Acid Detection Kit (Boehringer-Mannheim, Mannheim, Germany) as recommended by the manufacturer. Slides were finally washed in PBS and distilled water, and mounted in an aqueous medium.

To monitor the specificity of the reactions, the same samples were also treated omitting Dnase or hybridised with sense probes. Rabbit kidney was used as negative control.

\section{In situ reverse transcriptase polymerase chain reaction (IS-RT-PCR)}

With the aim to improve the results obtained by ISH, placental tissues were also subjected to an in situ reverse-transcriptase PCR protocol. The sections underwent the same pre-treatments employed with the ISH procedure. After the removal of DNA, RNA was reverse-transcribed into single strand cDNA, by using a solution containing $1 \mathrm{x}$ RT buffer, $5 \mathrm{mM}$ $\mathrm{MgCl}_{2}, 1 \mathrm{mM}$ dNTP, $0.2 \mu \mathrm{g} / \mu \mathrm{l}$ random examers, 2 U/ $\mu$ l RNase inhibitor, 2 U/ $\mu$ l Avian Mieloblastosis Virus (AMV) Reverse Transcriptase, RNase-free water up to $20 \mu \mathrm{l}$ each slide $\left(1 \mathrm{~h}\right.$ at $42^{\circ} \mathrm{C}$.).

The slides were taken off the capillary system for the in situ PCR. The amplification step was performed with the PCR Core Kit (Boehringer) as recommended by the manufacturer.

Primers used were designed to amplify a 229 bp portion of the en-JSRV GAG gene (sense 5'-GCT-
GCTTTGAGACCT TATCGAAA-3', antisense 5'-ATACTGCAGCTCGATGG CCAG-3'). Coverslips were added and sealed with Hybaid Easy Seal Starter Kit (Hybaid Ltd., Waldegrave, Teddington, Middlesex, UK) and the slides were then placed in an in situ thermal cycler (Hybaid Omnislide 2000). The amplification process consisted of one cycle at $94^{\circ} \mathrm{C}$ for $1 \mathrm{~min}$, followed by 30 cycles at $94^{\circ} \mathrm{C}$ for $45 \mathrm{sec}, 57^{\circ} \mathrm{C}$ for $1 \mathrm{~min}$, and at $72^{\circ} \mathrm{C}$ for $1 \mathrm{~min}$, with a final extension at $72^{\circ} \mathrm{C}$ for $2 \mathrm{~min}$. After completion of the PCR, the slides were re-transferred into the capillary slide-holder, washed thrice in PBS for $10 \mathrm{~min}$, post-fixed in $4 \%$ paraformaldeheyde and washed again in PBS. Prehybridisation, hybridisation and signal development were then performed as reported for the ISH procedure.

As reagent controls, the same samples were also treated omitting Dnase and/or the reverse transcription step. Rabbit kidney was used as negative control.

\section{RESULTS}

\section{ISH}

The RNA in situ hybridisation revealed a number of signals in various foetal and placental tissues.

With regard to the foetuses, positive reactions were observed within mesenteric lymph nodes, mostly located in the cytoplasm of small and medium-sized lymphocytes (Fig. 1). Similar results were observed in the medulla of the thymus lobules (Fig. 2), in which the chromogen stained large, swollen reticular epithelial elements (Fig. 3) and small lymphocytes (Fig. 4). A low number of epithelial bronchiolar cells in the lungs (Fig. 5) also showed positive areas.

The placentas revealed signals which were mainly located in the cytoplasm of elements belonging to the syncithium trophoblast (Fig. 6). The positive areas were regularly diffused throughout the samples.

All the tested tissues (lymph nodes, thymi, lungs and placentas) did not give any appreciable reaction when treated with Dnase and sense probes (Fig. 7); a labelling of the nuclei only appeared in the sections hybridised with sense probes and in which the Dnase step had been omitted (Fig. 8), 

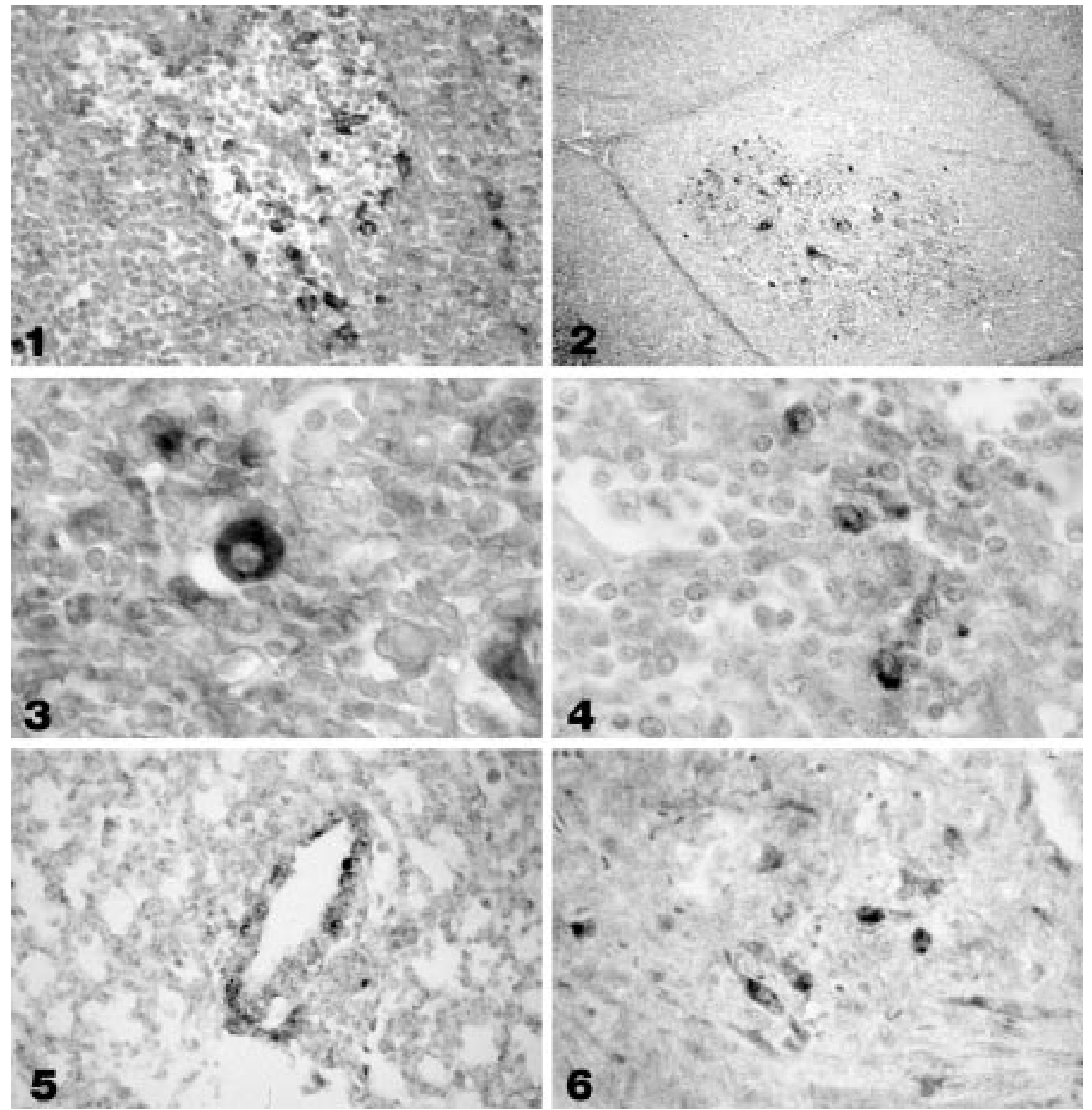

Fig. 1 - Ovine foetal mesenteric lymph node. ISH for enJSRV RNA. Labelling of small lymphocytes. Anti-Dig-AP-NBT system, x 25.

Fig. 2 - Ovine foetal thymus. Positive signals are present in the medulla. ISH, x 10.

Fig. 3 - Ovine foetal thymus. Positive reticular cell. ISH, x 100.

Fig. 4 - Ovine foetal thymus. Labelling of some lymphocytes. ISH, x 100.

Fig. 5 - Ovine foetal lung. Some bronchiolar cells show a weak positivity. ISH, x 25.

Fig. 6 - Ovine placenta. EnJSRV RNA is present in the syncithium trophoblast. ISH, x 40.

thus confirming the specificity of the tests. In the negative controls (rabbit kidneys) treated with antisense and sense probes and with or without Dnase, we could not observe any positive hybridocytochemical signal.

\section{IS-RT-PCR}

Strong reactions were observed within all the tested samples. A good number of cells showed intracytoplasmic signals. Longitudinal sections of the placental tissue revealed that en-JSRV RNA was 

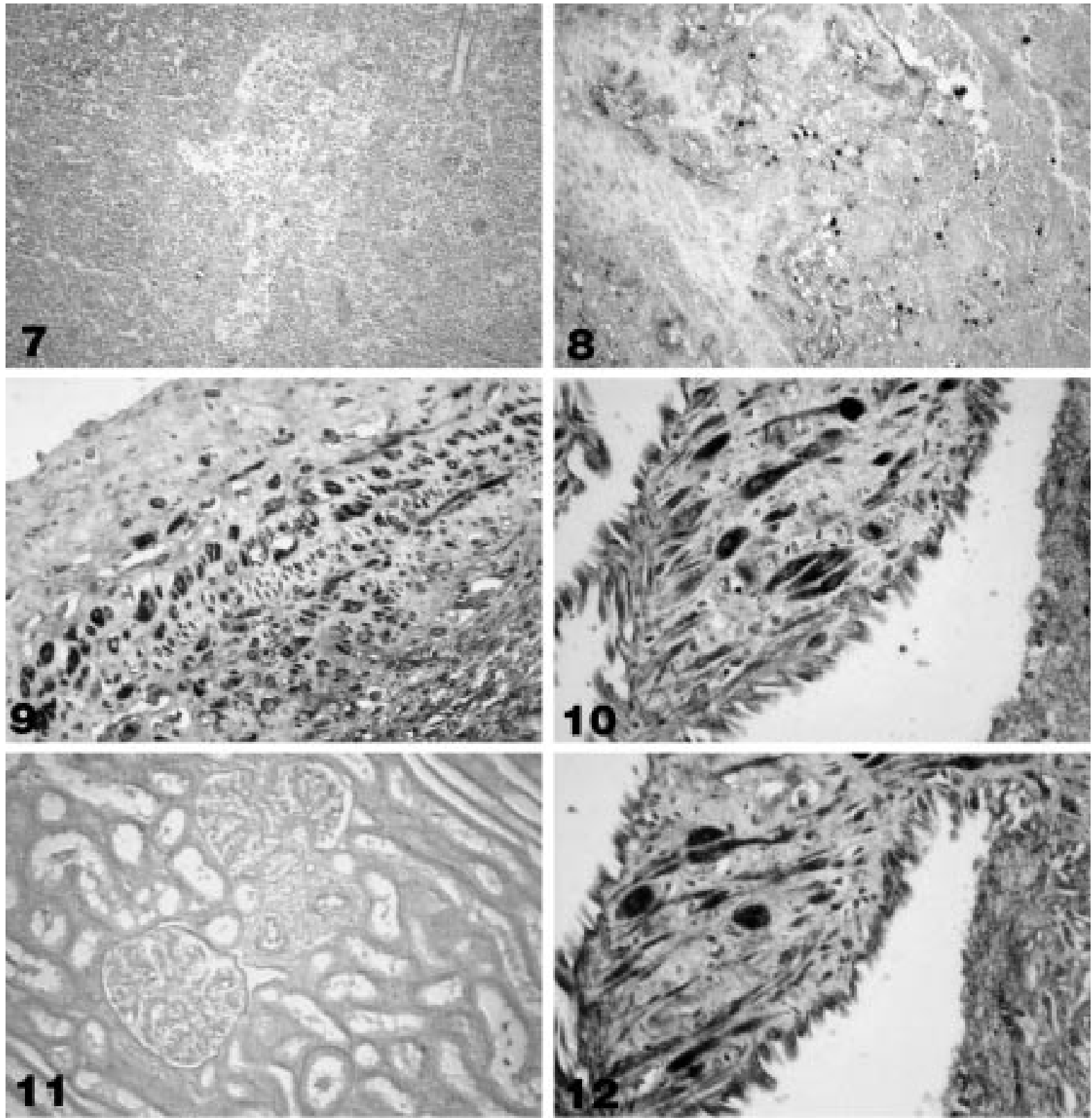

Fig. 7 - Ovine lymph node. Sense probe control. No appreciable reaction is present, ISH, x 10

Fig. 8 - Ovine placenta. Sense probe control. Omission of Dnase treatment: some nuclei are labelled (hybridisation of the integrated enJSRV proviral DNA). ISH, x 10.

Fig. 9 - Ovine placenta. IS-RT-PCR for enJSRV RNA. Longitudinal section reveals a positive labelling in maternal and foetal elements. IS-RT-PCR, x 25.

Fig. 10 - Ovine placenta. Cross section of villi shows positive foetal fibroblasts. IS-RT-PCR, x 40.

Fig. 11 - Rabbit kidney. Total Absence of signals. IS-RT-PCR, x 10.

Fig. 12 - Ovine placenta. Omission of Dnase treatment: labelling of the nuclei. IS-RT-PCR, x 40.

present in bi-nucleated cells and in hybrid and foetal elements (Fig. 9). Interestingly, cross sections of the villi clearly showed that foetal fibroblasts were strongly positive (Fig. 10). The rabbit kidney controls constantly gave negative results (Fig. 11), whilst in the sections in which the treatment with 
Dnase was omitted, the chromogen bonded to the nuclei, as a result of the hybridisation of the integrated enJSRV proviral DNA (Fig. 12). In the noRT controls we could observe a significant reduction of the signals; in practice, these samples showed positive reactions only in the cells which had been labelled with the ISH protocol.

\section{DISCUSSION}

This study was carried out to assess whether and where enJSRV is transcriptionally active in ovine foetus and placenta. This is the first report that conclusively associates the expression of the endogenous retrovirus with those tissues and that identifies some involved cells. In addition, we have developed a reliable IS-RT-PCR protocol which may be useful in further studies on the expression of the endogenous retrovirus.

Our primary objective was to verify the expression of enJSRV in organs which are critical for the development of the immune system. At the moment, little is known on the interaction of exogenous JSRV with the immune system of the host. OPC-affected sheep do not seem to have circulant antibodies towards JSRV (Ortin et al., 1998) and these data led to the speculation that enJSRV related antigens may be expressed during ontogeny. With this regard, the data we obtained are of interest, because positive signals were observed both in lymphatic and reticular epithelial elements located in the medulla of the thymic lobules. These results may therefore support the hypothesis that a tolerance state for retroviral products is established during foetal life and could partially explain the apparent absence of immunological reactions against the exogenous counterpart during naturally occurring infection. The enJSRV ability to produce viral RNA in lymphatic lineage is confirmed by the detection of labelled elements in mesenteric lymph nodes. A specific lympho-tropism had already been assessed for the exogenous JSRV, which is effectively able to establish a disseminated infection of the lymphoid tissue (Palmarini et al., 1996b, Holland et al., 1999). On the other hand, the signals we could observe in the foetal bronchiolar epithelia were very rare and weak. This finding matches the results obtained by Pal- marini et al. (2000b) on adult sheep tissues and is consistent with the general opinion that enJSRV does not have a strong specificity for lung tissue. In the placenta, positive results were detected in different cell types. They involved both maternal and foetal elements. The high RT-IS-PCR sensitivity revealed signals in a cell spectrum broader than that obtained by ISH alone, as the amplification of the target allowed the labelling of some large fibroblasts. The results obtained with the two different techniques may reflect the possibility that, in the placenta, only one or a few number of copies of enJSRV could undertake transcriptional activity at a single cell level (Palmarini et al., 2000b). Recently, it has been observed that in the pregnant ewes, the expression of enJSRV genes in the endometrial epithelia is correlated with the peripheral blood levels of progesterone and the presence of progesterone receptor in uterine epithelia, and may result from the activation of LTR promoters and enhancers stimulated by the hormone (Palmarini et al., 2001). By this point of view, the expression of enJSRV we observed in the placenta may be consistent with the hypothesis of a progesterone-induced activation, as this hormone is largely produced in situ until the latest stage of the ewe pregnancy.

Taken together, the data we obtained demonstrate that, in ovine foetus and placenta, enJSRV is largely expressed in a variety of cell types. The biological plasticity of enJSRV had already been demonstrated in adult sheep and it also reflects the behaviour of the exogenous counterpart. The tropisms of the endogenous and exogenous forms are however different and may be driven by the peculiarities of the LTR regions. This hypothesis is supported by the observation that, in JSRV, LTRs are preferentially active in type II pneumocytes and Clara cells, which represent the main targets of the retrovirus and the progenitors of the neoplastic cells (Palmarini et al., 2000a). With this regard, it has been speculated that JSRV became lung tropic during its evolution as an exogenous virus, but its ancestor (from which both enJSRV and JSRV originated) did not have a pulmonary tropism (Palmarini et al., 2000b). The immunological tolerance of the sheep induced by the expression of enJSRV during foetal life, may have facilitated the evolution of JSRV tropism. 


\section{ACKNOWLEDGMENTS}

Prof. E. Sanna and Dr. M.P. Sanna share primary authorship for this work. The Authors thank Prof. Massimo Palmarini from the Department of Medical Microbiology and Parasitology, College of Veterinary Medicine, University of Georgia, Athens, USA for the initial discussion leading to this work, for providing the sequence of hybridisation probes and for the critical review of the manuscript. This research has been supported by funds from the Italian Ministero dell'Università e della Ricerca and by a grant from the Università degli Studi di Sassari.

\section{REFERENCES}

Bai J., Zhu R.J., Stedman K., Cousens C., Carlson J., Sharp J. M., and DeMartini J.C.: Unique long terminal repeat U3 sequences distinguish exogenous jaagsiekte sheep retrovirus associated with ovine pulmonary carcinoma from endogenous loci in the sheep genome. J. Virol. 70, 3159-3168, 1996.

Best S., Le Tissier P., Towers G., and Stoye J. P.: Positional cloning of the mouse retrovirus restriction gene Fv1. Nature $382,826-829,1996$

Feuchter A., and Mager D.: Functional heterogeneity of a large family of human LTR-like promoters and enhancers. Nucleic Acids Res. 18, 1261-1270, 1990.

Feuchter, A., and Mager, D.: SV40 large T antigen trans-activates the long terminal repeats of a large family of human endogenous retrovirus-like sequences. Virology 187, 242 250, 1992.

Hecht,S.J., Carlson J.O., and Demartini J.C.: Analysis of a type D retroviral capsid gene expressed in ovine pulmonary carcinoma and present in both affected and unaffected sheep genomes. Virology 202, 480-484, 1994.

Hecht S.J., Stedman K.E., Carlson J.O., and DeMartini J.C. Distribution of endogenous type B and type D sheep retrovirus sequences in ungulates and other mammals. Proc. Natl. Acad. Sci. U.S.A 93, 3297-3302, 1996.

Herbst H., Sauter M., and Mueller-Lantzsch, N.: Expression of human endogenous retrovirus $\mathrm{K}$ elements in germ cell and trophoblastic tumors. Am. J. Pathol. 149, 1727-1735, 1996.

Holland M.J., Palmarini M., Garcia Goti M., Gonzalez L., Mckendrick I., De Las Heras M., and Sharp, J.M.: Jaagsiekte retrovirus is widely distributed both in $\mathrm{T}$ and $\mathrm{B}$ lymphocytes and in mononuclear phagocytes of sheep with naturally and experimentally acquired Pulmonary Adenomatosis. J. Virol. 73, 4004-4008, 1999.

Katsumata K.: Tissue-specific expression of human endogenous retrovirus mRNA and its regulation by cytokines in vitro. Hokkaido Igaku Zasshi; 72, 635-648, 1997.
Krieg A.M., Gause W.C., Gourley M.F., and Steinberg A.D.: A role for endogenous retroviral sequences in the regulation of lymphocyte activation. J. Immunol. 143, 2448-2451, 1989.

Larsson E., and Andersson G.: Beneficial role of human endogenous retroviruses: facts and hypotheses. Scand. J. Immunol. 48, 329-338, 1998.

Löwer R., Löwer J., and Kurth L.: The viruses in all of us: charachteristics and biological significance of human endogenous retrovirus sequences. Proc. Natl. Acad. Sci. USA 93, 5177-5184, 1996.

Maeda N., Palmarini M., Murgia C., and Fan, H.: Direct transformation of rodent fibroblasts by jaagsiekte sheep retrovirus DNA. Proc. Natl. Acad. Sci. USA 98, 44494454, 2001.

Nakagawa K., and Harrison L.C.: The potential roles of endogenous retroviruses in autoimmunity. Immunol. Rev., 152, 193-236, 1996

Ortin A., Minguijon E., Dewar P., Garcia M., Ferrer L.M., Palmarini M., Gonzalez L., Sharp J.M., and De las Heras, M.: Lack of a specific immune response against a recombinant capsid protein of Jaagsiekte sheep retrovirus in sheep and goats naturally affected by enzootic nasal tumour or sheep pulmonary adenomatosis. Vet. Immunol. Immunopathol. 61, 229-237, 1998.

Palmarini M., Dewar P., De Las Heras M., Inglis N.F., Dalziel R.G., and Sharp, J.M.: Epithelial tumour cells in the lungs of sheep with pulmonary adenomatosis are major sites of replication for jaagsiekte retrovirus. J. Gen. Virol. 76, 2731-2737, 1995.

Palmarini M., Cousens C., Dalziel R.G., Bai J., Stedman K., De Martini, J.C., and Sharp, J.M.: The exogenous form of jagsiekte retrovirus is specifically associated with a contagious lung cancer of sheep. J. Virol. 70, 1618-1623, 1996a.

Palmarini M., Holland M.J., Cousens C., Dalziel R.G., and Sharp, J.M.: Jaagsiekte retrovirus establishes a disseminated infection of the lymphoid tissues of sheep affected by pulmonary adenomatosis. J. Gen. Virol. 77, 2991-2998, $1996 b$

Palmarini M., Sharp J.M., De Las Heras M., and Fan, H.: Jaagsiekte sheep retrovirus is necessary and sufficient to induce a contagious lung cancer in sheep. J. Virol. 73, 6964$6972,1999$.

Palmarini M., Datta S., Omid R., Murgia C., and Fan, H.: The long terminal repeats of jaagsiekte sheep retrovirus (JSRV) are preferentially active in type II pneumocytes. J. Virol. 74, 5776-5787, 2000a

Palmarini M., Hallwirth C., York D., Murgia C., de Oliveira T., Spencer, T., and Fan, H.: Molecular cloning and functional analysis of three type D endogenous retroviruses of sheep reveal a different cell tropism from that of the highly related exogenous jaagsiekte sheep retrovirus. J. Virol. 74, 8065$8076,2000 b$. 
Palmarini M., Gray C.A., Carpenter K., Fan H., Bazer F.W., and Spencer T.E.: Expression of endogenous betaretroviruses in the ovine uterus: effects of neonatal age, estrous cycle, pregnancy, and progesterone. J. Virol. 75, 11319-1127, 2001.

Sanna M.P., Sanna E., De Las Heras M., Leoni A., Nieddu A.M., Pirino S., Sharp J.M., and Palmarini M.: Association of
Jaagsiekte Sheep Retrovirus with pulmonary carcinoma in sardinian moufflon (Ovis musimon). J. Comp. Pathol. 125, $145-152,2001$

Taruscio D., and Mantovani A.: Human endogenous retroviral sequences: possible role in reproductive physiopathology. Biol. Reprod. 59, 713-724, 1998. 